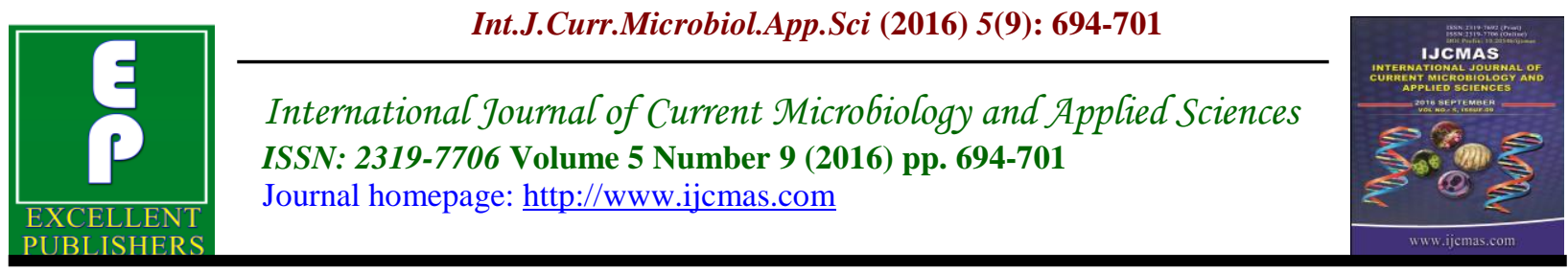

Original Research Article

http://dx.doi.org/10.20546/ijcmas.2016.509.080

\title{
Performance of Matrix-Assisted Laser Desorption Ionization-Time of Flight Mass Spectrometry for Routine Identification of Aerobic Gram Negative Bacteria Isolated in Zagazig University Hospital, Egypt
}

\author{
Ghada E. Amr* and Hoda A. Ebraheem \\ Department of Clinical Pathology, Faculty of Medicine, Zagazig University, Egypt \\ *Corresponding author
}

\section{Keywords}

Matrix-assisted laser desorption ionizationtime of flight mass spectrometry (MALDITOF MS), Gramnegative bacteria, identification.

Article Info Accepted: 25 August 2016 Available Online: 10 September 2016

\section{A B S T R A C T}

Accurate and rapid identification of bacteria is critical for the appropriate management of infections. Bacterial identification relies primarily on culture-based methodologies that requiring 24 hours for isolation and an additional 24 to 48 hours for species identification. Recently, Matrix-assisted laser desorption ionizationtime of flight mass spectrometry (MALDI-TOF MS) has been introduced in diagnostic microbiology laboratories as rapid, highly accurate and cost-effective method for routine identification of a wide range of microorganisms. To evaluate the analytical and practical performance of MALDI-TOF MS (VITEK MS) system in comparison to conventionalVitek2 system for identification of Gram-negative bacilli. A total of 100 aerobic Gram-negative bacilli isolates representing 10 genera and 14 species obtained from different hospital wards were analyzed. Unmatched results were further investigated by $16 s$ rRNA gene sequencing as a reference method. 88 isolates $(88 \%)$ had completely matched results by both systems, while12 isolates had unmatched results. VITEK MS identified all of isolates $(100 \%)$ to the genus level and $98 \%$ of isolates to the species level, while Vitek2 identified $97 \%$, and $90 \%$ to genus and species level respectively. VITEK MS also reduces the time and cost for routine identification of the studied isolates. Our result showed that VITEK MS is accurate, convenient, simple, rapid and cost saving technique for routine bacterial identification and it has a potential to replace the biochemical and other phenotypic systems for routine identification of Gramnegative bacteria in the clinical microbiology laboratory.

\section{Introduction}

The identification of bacterial and fungal species in clinical microbiology laboratories is mainly carried out according to phenotype characteristics, including identifications of culture media, colony morphology, Gramstain and various biochemical reactions (Carroll et al., 2007).
Most of these methods are laborious, time consuming and require long turnaround time to complete the whole identification process with high accuracy. Molecular methods, such as real-time PCR, gene sequencing and microarray analysis, are quick methods for bacterial and fungal identification, but they 
come at a very high cost and require highlytrained technicians, factors that limit their use in routine bacterial identification (Guo et al., 2014).

The development of matrix-assisted laser desorption ionization time-of-flight mass spectrometry (MALDI-TOF MS) for identification of microorganisms based on the comparison of sample mass spectral fingerprints that are unique protein signatures for each microorganism to reference mass spectral fingerprints. One or a set of characteristic peaks in the mass spectral fingerprints, which appear to be conserved for a certain type of microorganism, are used for the identification (Deng et al., 2014). MALDITOF MS has been reported to be a fast, reliable and cost-effective technique for routine application in clinical laboratories (Deng et al., 2010; Benagli et al., 2011).

The aim of this study is to evaluate the performance of MALDI-TOF MS system (VITEK MS) that was newly introduced in our laboratory in parallel with our routine phenotypic Vitek 2 system. We employed both systems to identify and analyze 100 aerobic Gram-negative bacilli that were routinely isolated at microbiology laboratory of Zagazig University Hospital. We have analyzed these organisms as it were the most common isolated organism in our hospital Intensive Care Unit (ICU) and it were most challenging from diagnostic and therapeutic standpoint.

\section{Methods}

A total of 100 isolates of aerobic Gramnegative bacilli were isolated from patients, who were tested during a period of two months (January and February 2015), that isolates were encompassed Enterobacteriaceae, non-fermentative Gram- negative bacilli isolated from 46 urine samples, 26 sputum samples, 11 pus samples, 14 positive blood cultures, 2 peritoneal fluid samples and one fecal samples. All isolates from different origins were recovered after culture on suitable media (blood agar, MacConeky agar, chocolate agar) and aerobic incubation. Growth of colonies was ascertained after overnight incubation by visual inspection. Blood cultures were done using Bact /ALERT FA culture bottles and incubated for 7 days in the automated Bact / AlERT system (Biomerieux. Inc, Durham, USA).

\section{Vitek 2 identification}

Vitek 2 instrument that used extensive biochemical panels (Biomerieux. Inc, Durham, USA) was loaded by Gramnegative $(\mathrm{GN})$ identification cards that were selected to run identification analysis of the different strains and were tested after adjustment of McFarland standard of 0.5 to 0.6 with bacterial inoculums.

\section{MALDI-TOF MS identification}

The strains were identified by MALDI-TOF MS using the VITEK MS system (Biomerieux. Inc, Durham, USA).Small amount of the freshly grown test isolates that were incubated for 24 hours were picked and smeared on the wells of disposable target slides to form thin layers of organisms, $1 \mu \mathrm{L}$ of VITEK MS CHCA matrix solution (cyano-4-hydroxycinnamic acid) was applied over the sample and airdried for 1 to $2 \mathrm{~min}$ at room temperature. The Escherichia coli ATCC 8739 strain was used as a calibrator and internal identification control. It was inoculated on the calibration spots of each acquisition group. The target slide with all prepared samples was then loaded into the VITEK MS system to acquire the mass spectra of 
whole bacterial cell protein. Finally, the mass spectra acquired for each sample were compared to the known mass spectra contained in the database and given a confidence score according to how close the acquired spectra matched those contained in the database. Peak matches that yielded identification results with confidence values exceeding 90\% were considered significant and displayed. Identification was done using a new database (v2.0) and MYLA software developed for in vitro diagnostic (IVD) use.

\section{Gene sequencing}

Discrepancies between VITEK MS system and Vitek 2 were investigated by $16 S r R N A$ gene sequencing (reference method) using FAST MicroSEQ ${ }^{\circledR} 500$ 16S rDNA Bacterial Identification Kit (Applied Biosystems, Carlsbad, CA) which comprises two kits: the FAST MicroSEQ® 50016S rDNA PCR Kit and the MicroSEQ ${ }^{\circledR 500} 16 \mathrm{~S}$ rDNA Sequencing Kit. DNA was extracted using the PrepMan ${ }^{\mathrm{TM}}$ Ultra Sample(Applied Biosystems, USA), 16S ribosomal RNA genes amplified then analyzed using MicroSEQ® ID Analysis Software that compared the sequence to the validated MicroSEQ ${ }^{\circledR}$ ID 16S rDNA 500 Library.

\section{Results and Discussion}

There were 100 isolates included in the study representing 10 genera and 14 species. Eighty eight isolates had the same matched identification results by both VITEK MS and Vitek 2(table 1). On the other hand 12 isolates had unmatched results by both systems that were further investigated and identified by $16 S r R N A$ gene sequencing that was used as a reference method (table2). The final identification by all the method used were 34 E.coli, 25 Klebsiella pneumoniae, 15 Acinetobacter bumanii, 6 Pseudomonas aeruiginosa, 4 Proteus vulgaris, 3 Proteus mirabilis, 2 Enterobacter aerogenes, one Enterobacter cloacae, 2 Klebsiella oxytoca, 3 Serratia liquefaciens, one Salmonella enterica, 2 Citrobacter braaki, one Citrobacter freundi, and one Burkholderia cepacia.

The results of sequencing showed that the errors made by VITEK MS were $0.0 \%$ at genus level and $2 \%$ at species level as it misidentified two isolates (Citrobacter braaki as Citrobacter freundii and Enterobacter cloacae as Enterobacter aerogenes), while the errors made by Vitek 2 at genus level were $3 \%$ (2 Serratiaand one Salmonella) and at species level were 10 $\%$ (2 Acinetobacter bumanii, 4 Proteus vulgaris, 2 Serratia liquefaciens, one Burkholderia cepacia and one Salmonella enterica) (table 2).

The turnaround time was dramatically reduced by VITEK MS as the time from picking bacterial colony to the time of receiving results for 16 isolates in parallel was done in 20 minutes, one isolate average time was 1.2 minutes in comparison minimum 480 to1440 minutes (8-24 hour) taken by Vitek 2.The cost of one sample identification by VITEK MS was \$2.0 while the cost of the same sample identified by Vitek 2 was \$ 6.0 (table 3).

The introduction of MALDI-TOF MS technology in clinical microbiology laboratories has potentially revolutionized the routine identification of microorganisms by introducing a simple, rapid, high throughput, and low-cost identification technique (6).

In the present study, MALDI-TOF MS (VITEK MS) showed satisfactory results of analytical and practical performance in identification of 100 pathogenic aerobic Gram-negative bacilli routinely isolated in our laboratory. We compared the 
performance VITEK MS to Vitek 2 system, any disagreement results of identification was resolved by performing $16 \mathrm{~S}$ rRNA gene amplification and sequencing on the discrepant isolates

Our results showed that VITEK MS offered a higher correct identification rate and a lower error rates at the species level when compared to Vitek 2. VITEK MS identified $100 \%$ of isolates to the genus level and $98 \%$ of isolates to the species level. The misidentified organisms by VITEK MS were different species in the correct genus. VITEK 2 identified $97 \%$, and $90 \%$ to genus and species level respectively.

This MALDI-TOF MS (VITEK MS) correct identification percentage are slightly higher than those reported by other authors such Van Veen et al., (2010) who reported that accuracy rates of MALDI-TOF MS identification were $97.1 \%$ and $92 \%$ to the genus and species levels, respectively. Also Spanu et al., (2011) stated that MALDI-TOF MS identified $99.60 \%$ of isolates to the genus level and $93.37 \%$ of isolates to the species level. Other more recent studies by Zhoe et al., (2014) reported that the overall performance of VITEK MS was significantly better than the conventional method both for correct genus and species identification $(94.7 \%, 85.2 \%$, respectively). Guo et al., (2014) reported that 99.6\% isolates were correctly identified at the genus level and $93.37 \%$ at the species level and other study byJamal et al., (2014) stated that VITEK MS correctly identified isolates to genus and species $(99.8 \%$ and $99.0 \%$, respectively). The difference between our study and these previous studies is most likely due to the different strains correct identification rates as their studies included yeast, anaerobic, aerobic Gram-positive and negative bacteria.
In this study Vitek 2 instrument that used extensive biochemical panels and considered most-used identification technique in modern microbiology laboratories made $3 \%$ errors at the genus level and $10 \%$ at species level that is considered to be satisfactory result. According to the Manual of Clinical Microbiology, automated identification systems should ideally achieve an accuracy rate of no less than $90 \%$ in comparison to reference method. This is in agreement with Jamal et al., (2014) who concluded that VITEK MS and Vitek 2 showed the ability to correctly identify Gram-negative bacteria.

However the results of Gram-negative bacilli identification by VITEK MS still higher than Vitek 2 as the results of sequencing showed that the errors made by VITEK MS at genus level were $0.0 \%$ and at species level were $2 \%$ while the errors made by Vitek 2 at genus level were $3 \%$ and at species level $10 \%$.

VITEK MS was misidentified Citrobacter braakii as Citrobacter freundii, and misidentified Enterobacter cloacae as Enterobacter aerogenes. This was in agreement with Richter et al., (2013) who reported that the use of the term Citrobacter freundii complex for $C$. freundii, $C$. youngae, $C$. braakii, $C$. werkmanii, and $C$. sedlakii may be the best approach to reporting mass spectrometry results for these species. In addition Enterobacter cloacae were more likely to have a genus- rather than species-level VITEK MS identification and concluded that VITEK MS identifications limited to the genus level were common for multiple Citrobacter and Enterobacter species.

In the present study used $16 \mathrm{~S}$ rRNA gene sequencing as a reference method as it is powerful diagnostic tools with high discriminatory power for species and genus 
level determinations, however in addition to its high cost, it often requires specialized instrumentation and dedicated laboratory space and staff. These factors limit its use in most laboratories making the use of automated instruments for the identification of bacterial isolates is the predominate solution.

We followed VITEK MS protocols that relying directly on whole-cell analysis from a fresh colony that are faster and easier to use than well-established culture-based platforms such as Vitek 2 system that its protocol need calibrating a bacterial suspension and loading the identification cards. The analysis of 16 isolates in parallel by VITEK MS was done in less than 20 minutes meaning that one bacterial almost done within 1.2 minute, this make turnaround time strongly favors the VITEK MS system in comparison to 8-24 hours (one day) by Vitek 2. Many authors concluded about the value of rapid bacterial identification by VITEK MS as Cherkaoui et al., (2010), Jamal et al., (2014) and Patel et al., (2015). Lower reagent and labor costs for organism identification is another benefit of VITEK MS as its cost of identification for one bacterial isolate was $\$ 2.0$ while the ordinary price of Vitek 2 identification was $\$$ 6.0. This means that VITEK MS can reduce the cost of identification reagents by 66.6\%. Many authors as Cherkaoui et al., (2010), Gaillot et al., (2011), Tan et al., (2012), Neville et al., (2011), Seng et al., (2009) also reported about the bacterial identification cost saving by MALDI-TOF MS.

In this study we found that the delivery of the bacterial identification result by VITEK MS to the physician one day earlier could save both the cost of one day use of empirical antibiotics (about 40 US \$ "calculated nowadays") and the time to start proper treatment and may lead to better outcome and shorter hospital stay.

Table.1 Matched identification results of aerobic Gram-negative bacilli by VITEK MS and Vitek2

\begin{tabular}{|l|c|}
\hline \multicolumn{1}{|c|}{ Identified Gram- negative bacilli } & Number of strains \\
\hline E.coli & No. \\
Klebsiella pneumoniae & 34 \\
Acinetobacter bumanii & 25 \\
Pseudomonas aeruiginosa & 13 \\
Proteus mirabilis & 6 \\
Enterobacter aerogenes & 3 \\
Klebsiella oxytoca & 2 \\
Citrobacter freundii & 2 \\
Serratia liquefaciens & 1 \\
Citrobacter braaki & 1 \\
Total & 1 \\
\hline
\end{tabular}


Table.2 Discrepancies and error in Vitek 2 and VITEK MS for the identification of aerobic Gram-negative bacilli

\begin{tabular}{|c|l|c|c|c|c|}
\hline \multirow{2}{*}{$\begin{array}{c}\text { Number } \\
\text { of strains } \\
(12)\end{array}$} & Gene sequencing results & \multicolumn{2}{|c|}{ VITEK MS correct results } & \multicolumn{2}{|c|}{ Vitek 2 correct results } \\
\cline { 3 - 6 } & & Genus & Species & Genus & Species \\
\hline 2 & Acinetobacter bumanii & 2 & 2 & 2 & 0 \\
1 & Citrobacter braakii & 1 & 0 & 1 & 1 \\
1 & Enterobacter cloacae & 1 & 0 & 1 & 1 \\
4 & Proteus vulgaris & 4 & 4 & 4 & 0 \\
2 & Serratia liquefaciens & 2 & 2 & 0 & 0 \\
1 & Burkhulderia cepacia & 1 & 1 & 1 & 0 \\
1 & Salmonella enterica & 1 & 1 & 0 & 0 \\
\hline
\end{tabular}

Table.3 Difference between time and cost of identification by MALDI-TOF MS \& VITEK 2

\begin{tabular}{|c|c|c|}
\hline Method of identification & Turnaround time (minutes) & Cost (US \$) \\
\hline VITEK 2 & $480-1440$ & 6 \\
\hline MALDI-TOF MS & 1.2 & 2 \\
\hline
\end{tabular}

Some authors also investigated the impact of this new technology that may optimize the choice of empirical antibiotic therapy as Clerc et al., (2013) who concluded that MALDI-TOF could become a vital step in guiding empirical treatment in cases of Gram-negative bacteremia.

Also Hodiamont et al., (2012) concluded that early MS identification of blood culture isolate is a valuable addition to traditional laboratory technique as it resulted in the recommendation to change antibiotic therapy in up to $29 \%$ of cases they studied.

An important limitation of this study was the relatively small number of Gram-negative bacilli isolates encountered during the time of the study which was due to economic reasons as we did not receive any research grant. Further evaluation study involving a large number of stock clinical isolates of these bacteria and other types of both Grampositive and negative bacteria is under way. Future research is needed to explore the clinical impact of early bacterial identification and time saving made by MALDI TOF (VITEK MS) on patient outcomes such as recovery, mortality and length of hospital stay.

In conclusion, MALDI-TOF MS is a rapid, accurate, simple, easy and cost saving technique for identification of clinically significant bacteria. These advantages and the continuous development of MALDITOF MS technology and its clinical database makes MALDI-TOF MS systems the technique of choice to replace the conventional methods for identification of the majority of Gram-negative bacilli isolated in the clinical microbiology laboratory.

\section{Abbreviations}

MALDI-TOF MS, Matrix-assisted laser desorption ionization-time of flight mass spectrometry; CHCA, cyano-4hydroxycinnamic acid. 


\section{References}

Benagli, C., Rossi, V., Dolina, M., Tonolla, M. Petrini, O. 2011. Matrix assisted laser desorption ionization-time of flight mass spectrometry for the identification of clinically relevant bacteria. PLoS One, 6(1): e16424.

Bizzini, A., Durussel, C., Bille, J., Greub, G. Prod hom, G. 2010. Performance of matrix-assisted laser desorption ionization-time of flight mass spectrometry for identification of bacterial strains routinely isolated in a clinical microbiology laboratory. $J$. Clin. Microbiol., 48: 1549-54.

Carroll, K.C., Einstein, M.P. 2007. Manual and automated systems for detection and identification of microorganisms, p.192-217. In P. R. Murray (ed.), Manual of clinical microbiology, 9th ed., vol. 1. ASM Press, Washington, DC.

Cherkaoui, A., Hibbs, J., Emonet, S., Tangomo, M., Girard, M., Francois, P., et al. 2010. Comparison of two matrix-assisted laser desorption ionization-time of flight mass spectrometry methods with conventional phenotypic identification for routine identification of bacteria to the species level. J. Clin. Microbiol., 48: 1169-1175.

Clerc, O., Prod'hom, G., Vogne, C., Bizzini, A., Calandra, T., Greub, G. 2013. Impact of matrix-ssisted laser desorption ionization time-of-flight mass spectrometry on the clinical management of patients with Gramnegative bacteremia: a prospective observational study. Clin. Infect. Dis., 56: 1101-7.

Deng, J., Fu, L., Wang, R., Yu, N., Ding, X., Jiang, L., et al. 2014. Comparison of MALDI- TOF MS, gene sequencing and Vitek 2 for identification of seventy three clinical isolates of enteropathogen. J. Thorac. Dis., 6(5): 539-544.

Gaillot, O., Blondiaux, N,. Loïez, C., Wallet, F., Lemaître, N., Herwegh, S., et al. 2011. Cost-effectiveness of switch to matrix-assisted laser desorption ionization-time of flight mass spectrometry for routine bacterial identification. J. Clin. Microbiol., 49: 4412.

Guo, L., Ye, L., \& Zhao, Q. 2014. Comparative study of MALDI-TOF MS and VITEK 2 in bacteria identification. J. Thorac. Dis., 6(5): 534-5.

Hodiamont, C.J., Gouliouris, T., Rozemeijer, W., et al. 2012. Direct identification of micro-organisms in positive blood cultures using MALDITOF MS: consequences for advice on antibiotic therapy. ECCMID London, poster 2322.

Jamal, W., John Albert, M. Vincent Rotimi. O. 2014. Real-time comparative evaluation of bioMerieux VITEK MS versus BrukerMicroflex MS, two matrix- assisted laser desorptionionization time-of-flight mass spectrometry systems, for identification of clinically significant bacteria. BMC Microbiol., 14: 289.

Martiny, D., Busson, L., Wybo, I., El Haj, R.A., Dediste, A. \& Vandenberg, O. 2012. Comparison of the Microflex LT and Vitek MS systems for routine identification of bacteria by matrixassisted laser desorption ionizationtime of flight mass spectrometry. $J$. Clin. Microbiol., 50: 1313-25.

Neville, S.A., Lecordier, A., Ziochos, H., Chater, M.J., Gosbell, I.B., Maley, M.W., et al. 2011. Utility of matrixassisted laser desorption ionizationtime of flight mass spectrometry following introduction for routine 
laboratory bacterial identification. $J$. Clin. Microbiol., 49: 2980-2984.

Patel, R. 2015. MALDI-TOF MS for the diagnosis of infectious diseases. Clin. Chem., 61(1): 100-11.

Richter, S.S., Sercia, L. Branda, J.A. 2013. Identification of Enterobacteriaceae by matrix-assisted laser desorption/ionization time-of-flight mass spectrometry using the VITEK MS system. Eur. J. Clin. Microbiol. Infect. Dis., 32(12): 1571-8.

Seng, P., Drancourt, M., Gouriet, F., La Scola, B., Fournier, P.E., Rolain, J.M. et al. 2009. Ongoing revolution in bacteriology: routine identification of bacteria by matrix-assisted laser desorption ionization time-of-flight mass spectrometry. Clin. Infect. Dis., 49: 543-551.

Spanu, T., De Carolis, E., Fiori, B., Sanguinetti, M., D'Inzeo, T., Fadda, G., et al. 2011. Evaluation of matrixassisted laser desorption ionizationtime-offlight mass spectrometry in comparison to rpoB gene sequencing for species identification of bloodstream infection staphylococcal isolates. Clin. Microbiol. Infect., 17: 44-49.
Tan, K.E., Ellis, B.C., Lee, R., Stamper, P.D., Zhang, S.X. \& Carroll, K.C. 2012. Prospective evaluation of a matrix-assisted laser desorption ionization-time of flight mass spectrometry system in a hospital clinical microbiology laboratory for identification of bacteria and yeasts: a bench-by-bench study for assessing the impact on time to identification and cost-effectiveness. J. Clin. Microbiol., 50: 3301-3308.

Van Veen, S.Q., Claas, E.C.J. \& Kuijper, E.J. 2010. High throughput identification of bacteria and yeast by matrix-assisted laser desorption ionisation time of flight mass spectrometry in conventional medical microbiology laboratories. J. Clin. Microbiol., 48(3): 900-907.

Zhou, C., Hu, B., Zhang, X., Huang, S., Shan, Y. \& Ye, X. 2014. The value of matrix-assisted laser desorption/ionization time-of- flight mass spectrometry in identifying clinically relevant bacteria: a comparison with automated microbiology system. J. Thorac. Dis., 6(5): 545-552.

\section{How to cite this article:}

Ghada E. Amr and Hoda A. Ebraheem. 2016. Performance of Matrix-Assisted Laser Desorption Ionization-Time of Flight Mass Spectrometry for Routine Identification of Aerobic Gram Negative Bacteria Isolated in Zagazig University Hospital, Egypt. Int.J.Curr.Microbiol.App.Sci. 5(9): 694-701. doi: http://dx.doi.org/10.20546/ijcmas.2016.509.080 